\title{
Cues for Haptic Perception of Compliance
}

\author{
Wouter M. Bergmann Tiest and Astrid M.L. Kappers
}

\begin{abstract}
For the perception of the hardness of compliant materials, several cues are available. In this paper, the relative roles of force/displacement and surface deformation cues are investigated. We have measured discrimination thresholds with silicone rubber stimuli of differing thickness and compliance. Also, the influence of the finger span is assessed. When compliance is expressed as the Young's modulus, the thresholds in the different conditions follow Weber's law with a Weber fraction of 15 percent. When the surface deformation cue was removed, thresholds more than trebled. Under the assumption of optimal cue combination, this suggests that a large fraction of the information comes from the surface deformation cue. Using a matching experiment, we found that differences in object thickness are correctly taken into account. When cues appear to contradict each other, the conflict is resolved by means of a compromise.
\end{abstract}

Index Terms-Compliance, hardness, softness, psychophysics, haptic perception, touch.

\section{INTRODUCTION}

A $\mathrm{N}$ important part of haptic perception is interacting A with compliant materials. This type of perception is uniquely suited for judging, e.g., the ripeness of fruits, the air pressure in bicycle tires, or the quality of a mattress. It is, therefore, not surprising that there has been quite some attention to the subject of haptic perception of compliance, softness, or hardness, e.g., [1], [2], [3], [4], [5], [6], [7], [8].

The compliance of an object is determined by the elasticity of the material it is made of. The physical property of elasticity of a material is usually expressed as its Young's modulus $(Y)$, defined as the ratio between stress and strain

$$
Y=\frac{F / A}{\Delta l / l} \text {. }
$$

Stress is the force per unit area (pressure) $F / A$ that is applied to the object. Strain is the relative deformation $\Delta l / l$ that is the result. The unit is the same as that of pressure, Pascal (Pa). For "linear" materials, the Young's modulus is a property of the material, independent of the object's size or the amount of applied force.

The compliance of an object can also be expressed as its stiffness $(k)$, the ratio between applied force and displacement, in units of $\mathrm{N} / \mathrm{mm}$

$$
k=\frac{F}{\Delta l} .
$$

Stiffness is not independent of object thickness or the area of applied force. Stiffness is thus not a material property, but a property of the specific object and the way it is being touched. It is an unanswered question whether the haptic system takes these aspects into account when assessing the

- The authors are with the Physics of Man, Utrecht University, Padualaan 8, $3584 \mathrm{CH}$ Utrecht, The Netherlands.

E-mail: \{w.m.bergmanntiest, a.m.l.kappers\}@uu.nl.

Manuscript received 29 May 2008; revised 1 Feb. 2009; accepted 6 Apr. 2009; published online 27 Apr. 2009.

Recommended for acceptance by L. Jones.

For information on obtaining reprints of this article, please send e-mail to: toh@computer.org, and reference IEEECS Log Number TH-2008-05-0037.

Digital Object Identifier no. 10.1109/ToH.2009.16.

1939-1412/09/\$25.00 (C) 2009 IEEE hardness of a material. By "hardness" or "softness," we mean the subjective percept that people have when they handle compliant materials. It is important to make a distinction between that percept and stiffness or Young's modulus, which are physical measures for compliance.

It is known that the relationship between stiffness and subjective hardness is nonlinear, and can be described by a power law up to a certain upper limit [1]. Below this limit, materials can be discriminated if their stiffnesses differ by more than the discrimination threshold. This threshold has been measured to be around 13 percent, ${ }^{1}$ when the physical compliance measurement was done by compressing the stimuli between flat surfaces [2], and around 25 percent, when the physical compliance measurement was done by compressing the stimuli between a flat surface and a 1-inch diameter spherical surface [4]. Extensive research into softness discrimination was done by Tan et al. [5], but only with the fingers touching nondeformable surfaces. Therefore, those results cannot be generalized to a wider context. Srinivasan and LaMotte [6] showed that for the discrimination of softness, tactile (cutaneous) information is both necessary and sufficient. With an anesthetized finger, without cutaneous information, subjects could not discriminate between rubber specimens differing by a factor of 1.9 in stiffness. With passive touch, without kinesthetic force and displacement information available, subjects could discriminate between specimens of different compliance, using the surface deformation as a cue. However, in experiments with objects without a deformable surface, kinesthetic force and displacement information was necessary in addition to cutaneous force information. Experiments in which stimuli were actively palpated with a tool have also shown that force and displacement information can be used to discriminate softness [7]. Thus, both the ratio between force and displacement and the deformation of the touched surface provide information for the perception of softness. However, the relative importance of these cues has

1. The paper reports "percentage correct" versus the logarithm of the percentage of hardness difference $\Delta$. The curve crosses the 84 percent level at $\log \Delta \approx 1.1$, yielding $\Delta \approx 13$ percent. 
not yet been quantified. To measure this is one of the objectives of the present paper.

In virtual environments, with only force and displacement information available, a quantity called "rate-hardness" seems to be better correlated with hardness perception than stiffness [9]. However, with real materials, this does not seem to play a role [8], so we will not consider this phenomenon here. To provide surface deformation information in a virtual environment, a contact area spread rate display has been proposed [10]. This led to an improvement of about a factor of 2 in softness discrimination, but it is unknown if a device like this can truly capture the interaction between a finger and a deformable surface. We will compare the results from that study to ours. It has been shown that hardness perception is not a purely haptic process, but also has a visual component. The perceived stiffness of springs in a virtual environment that were visually and haptically incongruent, was greatly influenced by the visual presentation [11]. This effect has been used to successfully simulate haptic feedback when no force or displacement display was present at all [12]. This shows that visual displacement information also can be combined with cutaneous force information to form a percept of stiffness.

The question that comes to mind is: how do people arrive at their perception of hardness? Can they perceive the hardness of materials directly, i.e., get a direct impression of the compliance of an object, without the need to perform (subconscious) calculations involving forces and finger positions? If hardness is perceived directly, the accuracy of the perception should be independent of the size or shape of the object. For the indirect way of hardness perception, the accuracy should depend on the accuracy with which force and finger displacements are perceived. If varying these quantities does not affect hardness perception (i.e., discrimination thresholds do not change), then we can say that hardness is perceived directly and not through these separate quantities. The accuracy of force perception roughly follows Weber's law with a Weber fraction of $\sim 7$ percent [13]. The accuracy of displacement perception, in turn, depends on the accuracy of length perception between thumb and index finger, which has been found to violate Weber's law but does increase monotonically with reference length [14]. Does object size (and therefore, required finger span) influence the accuracy of hardness perception if it is based on the force/ displacement ratio? For an object of the same material but twice the thickness as another, half the amount of force is needed for the same displacement. After all, there is twice the amount of elastic material present between the fingers. Due to the constant Weber fraction, when the applied force is halved, the accuracy with which this force can be perceived gets better by a factor of $\sim 2$. At the same time, because the finger span has doubled, the accuracy of displacement perception gets worse by a similar factor. The net effect on the accuracy of hardness perception will be negligible. In order to study the way hardness is perceived (directly or indirectly), the factors of force and finger span should be decoupled in some way. To make this possible, a stimulus set has been designed which is described in the next section. In the sections that follow, three experiments making use of this stimulus set are described.
In the first experiment, the role of finger spread in the perception of compliance of real materials is investigated. In Experiment 2, the role of the surface deformation cue is assessed by completely removing this cue. Finally, in Experiment 3, confirmation of the findings from the first two experiments is sought by asking subjects to discriminate between the different types of stimuli.

\section{General Methods}

\subsection{Stimuli}

Stimuli of differing hardness were created by mixing two different types of silicone rubber: Wacker Elastosil M 4470 and 4500 . These are pourable silicone rubbers that vulcanize at room temperature under addition of a catalyst. Wacker Elastosil M 4470 is rather hard (Shore A 60), while 4500 is quite soft (Shore A 14). By mixing the two kinds, intermediate compliances were attained. In calculating the necessary masses of the two parts, the difference in their densities was taken into account. Twenty-three different mixing ratios were used, encompassing the whole range of compliance given by the two extremes that were the unmixed rubbers. There were three subranges of nine compliances (with a slight overlap) that constituted the soft, medium, and hard stimulus sets. In each compliance range, there was one reference stimulus, located in the center of the range, and eight test stimuli (four on either side). Based on pilot experiments, the compliance of the test stimuli was chosen in such a way that the spacing was the most dense close to the reference stimulus, and less dense further away from the reference stimulus, so that it was optimally suited for measuring precise psychometric curves. The mixtures were poured in cylindrical molds with a diameter of $41 \mathrm{~mm}$. Three different types of stimuli were fabricated: a set of 23 stimuli with a thickness of $20 \mathrm{~mm}$ ("thin"), a set of 23 with a thickness of $40 \mathrm{~mm}$ ("thick"), and a set of 23 that consisted of a 20-mm-thick disc of rigid synthetic material, with $10 \mathrm{~mm}$ of silicone rubber on either side ("sandwich"). This last type has the same total thickness as the "thick" type, but contains the same amount of compliant material as the "thin" type. In this way, an increase in thickness (and therefore, finger spread when grasping the stimulus) could be decoupled from an increase in the amount of compliant material between the fingers. Thus, for the "sandwich," the Young's modulus is the same as for the "thick" stimulus made of the same material, but the stiffness is different. A more detailed description of the fabrication procedure can be found in [15].

The compliance of the stimuli was characterized by means of an Instron 5542 Universal Material Testing machine. This machine measures force and displacement while the material is being compressed. Measurements for all 69 stimuli were performed in two ways: with the force applied with flat surfaces that had the same area as the stimuli, and with two cylindrical probes with a cross section of $100 \mathrm{~mm}^{2}$. This surface area was chosen to be similar to the contact area of fingers contacting the stimulus in a pinch grasp. The measurements with the small probes were performed with all three stimulus types. The measurements with the flat surfaces were only performed with the "thin" and "thick" types, because these measurements were to be used to calculate the Young's modulus of the materials. Force increasing at a constant rate of $1 \mathrm{~N} / \mathrm{s}$, from 0 to $20 \mathrm{~N}$ 


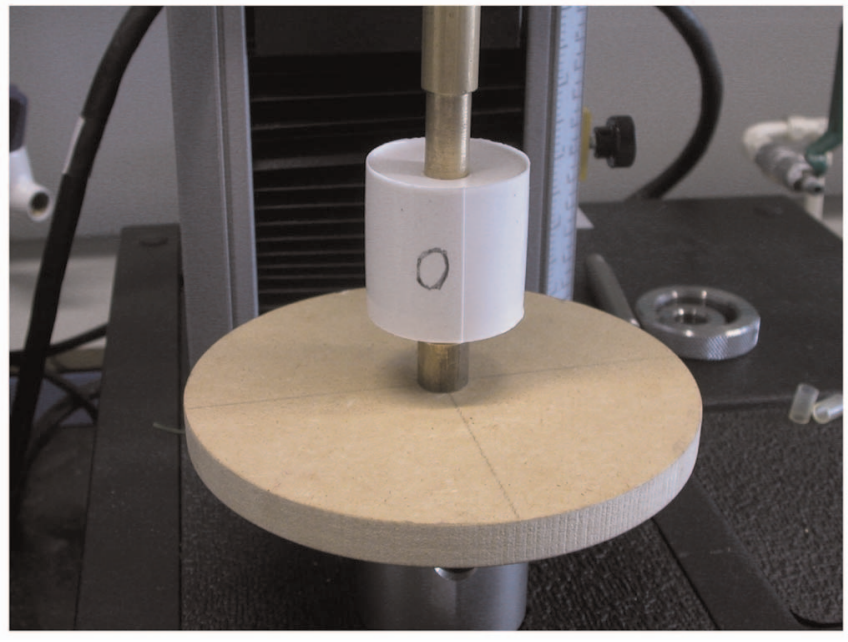

Fig. 1. Photograph of a stimulus being compressed between two probes

for the probes and 0 to $50 \mathrm{~N}$ for the flat surfaces, was applied, and measurements were taken at intervals of 1 N. A higher force range was used for the flat surfaces because the force is spread out over a larger area. The stimulus was positioned such that the probes indented the center of the stimuli. The sides of the stimuli were not restricted from expanding outward during the measurements. A photograph of a measurement in progress is shown in Fig. 1.

The measurements resulted in force versus displacement profiles for all stimuli. Examples for the three stimulus types are shown in Fig. 2. To determine the slope of these profiles, a straight line was fitted to the measurements. For the large contact area surfaces, there were some nonlinearities visible in the low-force range for some of the stimuli. These are probably due to the flat surfaces initially settling on the stimulus while the force increased. To get a fair estimate of the stimulus stiffness, the fit for the measurements with the flat surfaces was made to the data between 20 and 50 N. For the small contact area probes, the range of 1-20 N was used because this is typically the range of force used when squeezing. Each stimulus was measured five times for both ways of compressing to get an idea of the repeatability of the

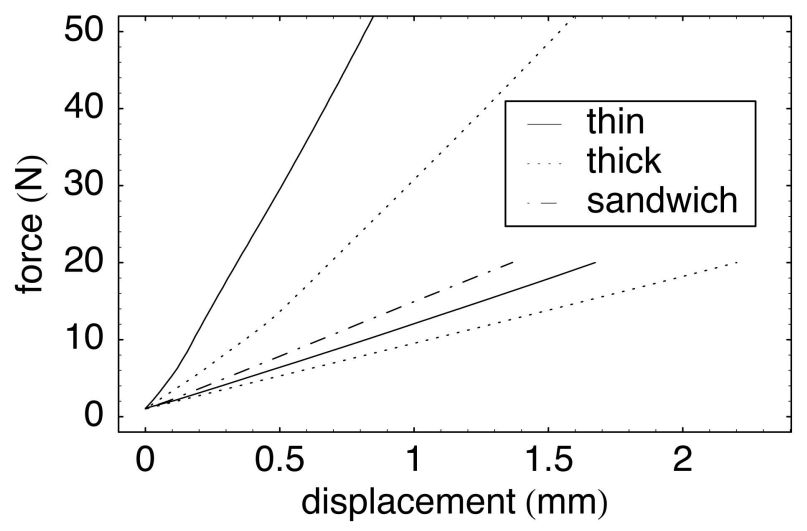

Fig. 2. Examples of force versus displacement profiles for the three stimulus types. The steep curves on the left going up to $50 \mathrm{~N}$ are for the flat surfaces, while the other curves are for the $100 \mathrm{~mm}^{2}$ cylindrical probes.

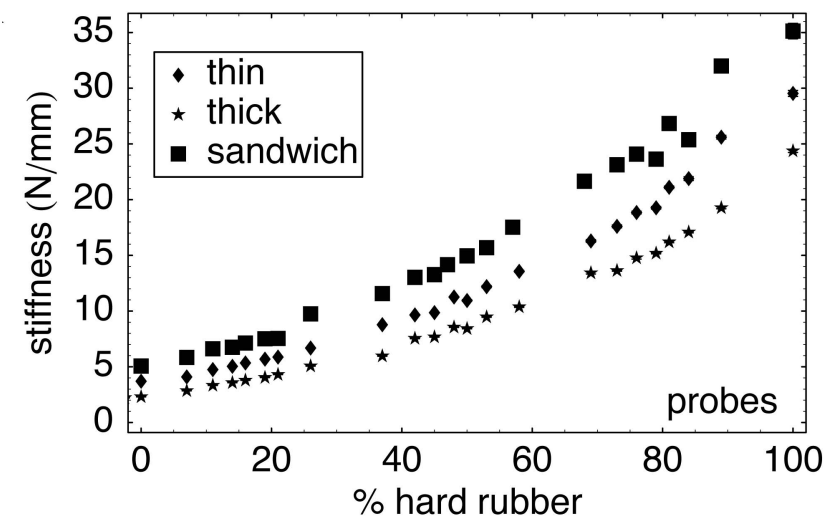

(a)

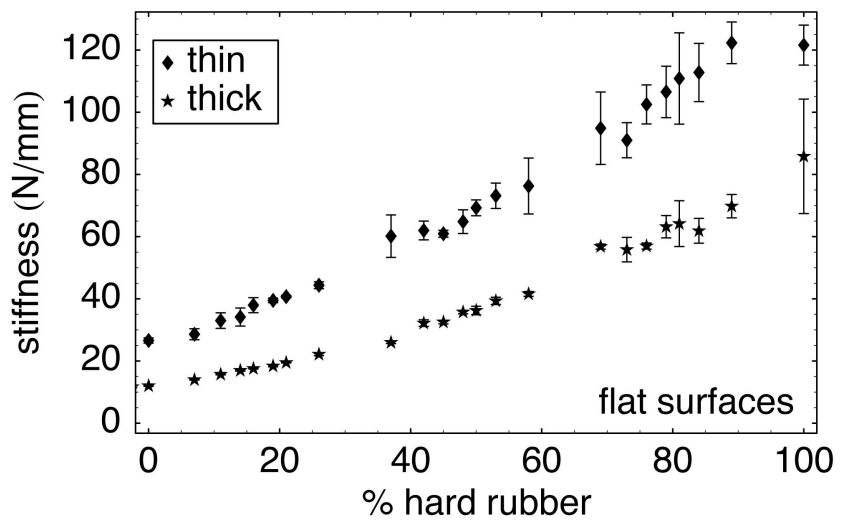

(b)

Fig. 3. Averages of the fitted slopes of the force versus displacement profiles as a function of the mass fraction of the hard rubber component for the different stimulus types, as measured with the (a) $100 \mathrm{~mm}^{2}$ probes and the (b) flat surfaces. For each of the stimulus types, the three hardness subsets can be distinguished by the grouping of the points. The error bars indicate the standard deviation of the five measurements.

measurements. In Fig. 3, the averages of the fitted slopes for the three sets of stimuli are plotted as a function of the mass percentage of the hard rubber component. For the measurements with the small probes (Fig. 3a), the error bars are generally smaller than the plot symbols, indicating a high degree of repeatability. For the measurements with the flat surfaces (Fig. 3b), in particular for the hardest stimuli, the spread is larger. As can be seen in the figure, the measured stiffness does not seem to increase monotonically with the percentage of hard rubber for all stimuli. This is surprising because the weighing and mixing of the components were done to a very high standard. However, since the measured values are used for the analysis, this should not affect the outcome of the psychophysical experiments. The measurements done with the flat surfaces on the "thin" and "thick" stimuli were used to calculate the Young's modulus of the materials. The measured slopes were divided by the surface area $\left(13.2 \mathrm{~cm}^{2}\right)$ and multiplied by the stimulus thickness (20 or $40 \mathrm{~mm}$, respectively). The resulting Young's moduli ranged between 0.38 and $2.2 \mathrm{MPa}$.

\subsection{Procedure}

The experiments conducted with the silicone rubber stimuli were two-alternative forced-choice discrimination experiments. In each trial, the blindfolded subject was presented 
with a test and a reference stimulus. These were placed in front of the subject, one in front of the other. Ridges on the table prevented the cylindrical stimuli from rolling to the side. The subject could comfortably grasp the stimuli, one at a time, between thumb and forefinger of the dominant hand and was asked to report which one felt harder. The subject could go back and forth between test and reference stimulus as often as desired. For each reference stimulus, there were eight test stimuli and each comparison between test and reference was made 10 times. The ordering of the trials was randomized and the positions of the test and reference stimuli were counterbalanced.

\subsection{Analysis}

The resulting data represented the number of times that a test stimulus was felt to be harder than the reference stimulus, as a function of the difference in physical hardness between the test and the reference. To this data, a cumulative Gaussian curve of the form $y=5 \operatorname{erf}(x / \sqrt{2} \sigma)+5$ was fitted. The width $\sigma$ of the cumulative Gaussian corresponds to the 84 percent level and is a measure of the discrimination threshold.

\section{EXPERIMENT 1: HARDNESS AND THICKNESS}

The purpose of the first experiment was twofold: to determine the dependence of the discrimination threshold on compliance, and to determine the influence of finger spread on the discrimination threshold. For the first purpose, the three compliance ranges soft, medium, and hard were used. For the second purpose, the three stimulus configurations "thin," "thick," and "sandwich" were used. Using those stimuli, the question might be answered whether the human haptic system can assess compliance (Young's modulus) as a material property, independent of object size (using information from the surface deformation), or whether it just uses the ratio between force and displacement. In the former case, a "thin" and "thick" stimulus of the same material should yield the same thresholds, since the material properties are the same even though the dimensions are not. In the latter case, a "thick" stimulus requires less force for the same indentation than a "thin" stimulus of the same material. If the accuracy of force perception is related to the magnitude of the force, then the smaller force for the "thick" stimulus is more accurately perceived. However, grasping the "thick" stimulus also requires a larger finger spread. If the accuracy of perception of changes in the finger distance is dependent on the magnitude of this distance, then the amount of indentation for the "thick" stimulus is less accurately perceived than for the "thin" stimulus. These two effects might cancel out, and we would still be unable to distinguish between the directperception hypothesis and the force/displacement-ratio hypothesis. But with the "sandwich" stimulus, the required force for a specific indentation would be similar to the "thin" stimulus (since they contain the same amount of compliant material), but the finger spread is the same as the "thick" stimulus. Therefore, the accuracy of force perception would not change much, while the accuracy of displacement perception would be worse than with the "thin" stimulus. So, if force/displacement information contributed to the

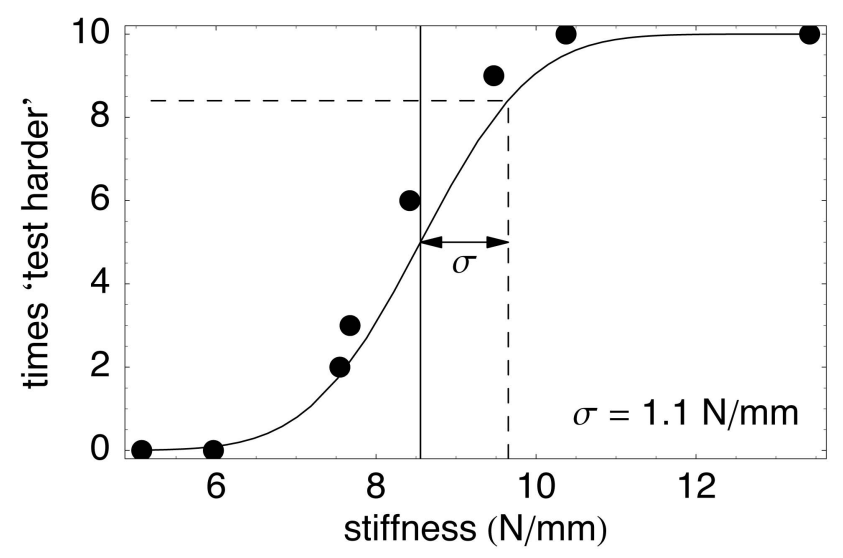

Fig. 4. Example of a psychometric curve with fitted function for the medium compliance range and thick stimulus configuration, with the stimulus compliance expressed as the stiffness as measured with the small probes. The solid vertical line indicates the stiffness of the reference stimulus. The dashed line indicates the position of the 84 percent level.

perception, we would expect a higher discrimination threshold in the "sandwich" case compared to the "thin" case.

\subsection{Method}

\subsubsection{Subjects}

Eight subjects (three male) were paid to participate. They ranged in age between 18 and 27 years. None had any known hand defects and all were right-handed according to Coren's test [16].

\subsubsection{Procedure}

The experiment was conducted according to the general methods described above. All three compliance ranges and all stimulus configurations were used, totaling 720 trials per subject. These were performed in several sessions of about 50 minutes each. Subjects had a maximum of two sessions per day and at least 45 minutes of rest between sessions. The trials were blocked according to stimulus type, but the compliances were mixed. For the ordering of the blocks, all six possible permutations were used, two of which were used twice. The total time taken ranged from 194 to 258 minutes per subject.

\subsection{Results}

The analysis resulted in nine psychometric curves per subject. In Fig. 4, an example is shown. In this case, the compliance is expressed as the slope (in $\mathrm{N} / \mathrm{mm}$ ) of the force/ displacement characteristic measured with the small probes. In Fig. 5, the thresholds for all stimulus configurations are shown as a function of reference compliance, averaged over subjects, for compliance expressed as (a) stiffness measured using the finger-sized probes, and (b) expressed as the Young's modulus calculated from the measurements with the flat surfaces on the "thin" and "thick" stimuli. For the analysis in terms of the Young's Modulus, for three of the 72 psychometric curves, the fitting procedure failed to yield a good fit, resulting in highly unlikely threshold values. Each problem occurred with a different subject. These three values were excluded from the data set. For the analysis in terms of stiffness, there was no problem with any of the 72 fits. 


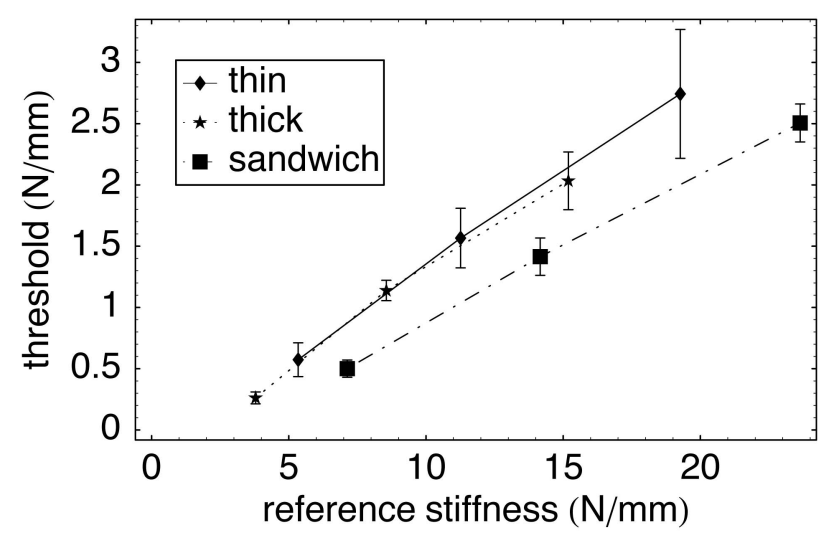

(a)

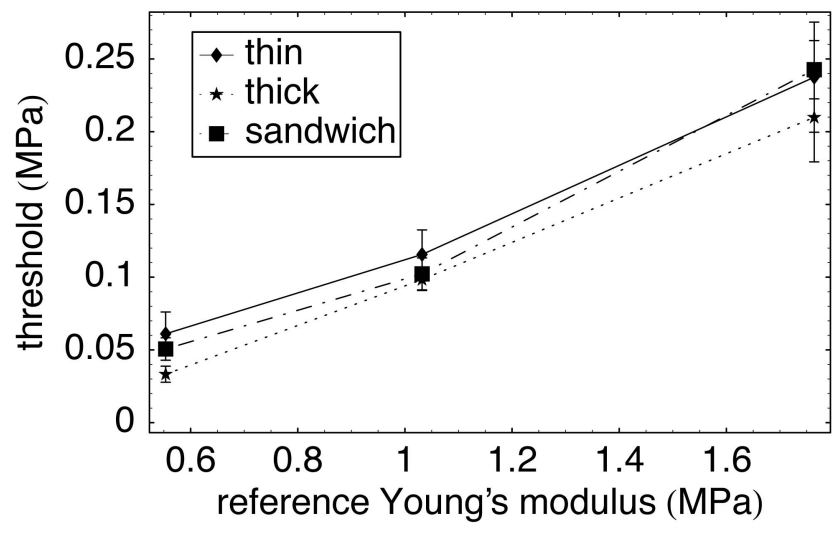

(b)

Fig. 5. Discrimination thresholds for the three stimulus configurations as a function of reference compliance, averaged over subjects. The error bars indicate the standard error of the mean. (a) Stiffness measured with finger-sized probes and (b) compliance expressed as the Young's modulus.

In Fig. 5a, the thresholds for the "thick" and "thin" stimuli lie approximately on the same straight line, while for the "sandwich" stimuli, the thresholds are lower. When the compliance is expressed as the Young's modulus (Fig. 5b), the thresholds for all three stimulus configurations appear to coincide. A 3 (stimulus configuration) $\times 3$ (compliance range) repeated-measures Analysis of Variance (ANOVA) on the stiffness thresholds showed a significant effect of compliance $\left(F_{2,14}=49, p=4.7 \times 10^{-7}\right)$ and of stimulus type $\left(F_{2,14}=4.2, p=0.038\right)$. For the Young's modulus thresholds, the effect of compliance was significant $\left(F_{2,8}=45, p=4.4 \times 10^{-5}\right)$, but the effect of stimulus configuration was not $\left(F_{2,8}=0.66, p=0.55\right)$. This means that when expressed in terms of Young's modulus, the thresholds are essentially independent of stimulus configuration. The slope of the straight line fitted to the thresholds in terms of Young's modulus for all stimulus configurations together, is $0.15 \pm 0.05\left(R^{2}=0.69\right)$. The reported standard deviation of 0.05 is the spread in the slopes of the individual subjects. The average slope of 0.15 may be interpreted as the Weber fraction for compliance discrimination in terms of Young's modulus.

Straight lines have also been fitted to the thresholds in terms of stiffness as measured with the small probes, shown in Fig. 5a. The slopes are $0.16 \pm 0.11\left(R^{2}=0.999\right), 0.15 \pm$ $0.06\left(R^{2}=0.99\right)$, and $0.12 \pm 0.03\left(R^{2}=0.999\right)$, for the "thin," "thick," and "sandwich" stimuli, respectively.

\subsection{Discussion}

The value of 0.15 for the Weber fraction found in this experiment is similar to what was found by Scott-Blair and Coppen [2], [3] $(\sim 0.13)$, but considerably smaller than the value of $\sim 0.3$ reported by Freyberger and Färber [4]. The compliance ranges in that and the present experiment are quite similar, so that cannot be an explanation for the discrepancy. The fact that in [4] the stimuli were blocks compared to the cylinders of the present experiment also does not seem able to explain the difference. A possible reason for the better performance in the present experiment could be that subjects were not restricted by a time limit and could feel the test and reference stimuli as often as they liked. In this way, the values found here might be more indicative of the true limit of human performance in ideal circumstances, and should be interpreted as a lower limit.

It is difficult to compare these results with those of Srinivasan and LaMotte [6], because they do not provide precise discrimination thresholds. In the experiment with the contact area spread rate display, an average just noticeable difference of about 26 percent of the stimulus intensity was reported [10]. The fact that in the present experiment a much lower Weber fraction was found, indicates that this display, although it provides more information than just force and displacement, still cannot produce an experience equal to direct contact with a real stimulus.

If the perception of hardness was based on the ratio between force and displacement, which is the definition of stiffness, one would expect a higher discrimination threshold in terms of stiffness for the "thick" stimulus compared to the "thin" stimulus, given the fact that the accuracy of displacement perception scales with finger spread [14]. That is to say, if we have a "thin" and a "thick" stimulus of equal stiffness as objectively measured, which are squeezed with identical force, then the resulting finger displacement is also the same. At the same time, the finger spread with the "thick" stimulus is twice as big as with the "thin" stimulus. Therefore, the accuracy with which the finger displacement can be perceived is worse with the "thick" stimulus. If the perception of hardness were based on calculating the ratio between force and displacement, then the accuracy of this hardness perception would be worse in the case of the "thick" stimulus. Similarly, a "thick" and a "sandwich" stimulus of equal stiffness would have the same discrimination threshold in terms of stiffness, since both force and displacement can be assessed with equal accuracy. Clearly, this is not confirmed by the experiment, as is shown by the fact that the discrimination thresholds for "thin" and "thick" fall on the same line in Fig. 5a, while those for the "sandwich" type are lower. This suggests that the subjects do not assess hardness by calculating the ratio between force and displacement or by estimating the stiffness, but are able to perceive the stimulus hardness by means of other cues, for example, the shape of the deformation of the stimulus surface. Those cues are more related to the compliance of the material, independent of the shape or size of the object. This is in line with what is seen in Fig. 5b, in which the data are 
presented in terms of the material's Young's modulus: in this case, there is no significant difference between the three stimulus types. This suggests that the hardness of the material itself is perceived directly, independent of finger spread. At the same time, it might be that the force/ displacement ratio still plays a small role, although this did not result in a significant difference between the stimulus types. To quantitatively assess the relative importance of the different cues, Experiment 2 was performed.

\section{Experiment 2: No Surface Deformation}

The motivation behind Experiment 2 was to investigate the contribution of the cue of surface deformation to the perception of hardness. By this cue, we mean the information about the compliance of an object that is not available when the object has a rigid surface, compared to when the object has a deformable surface. When a compliant stimulus is squeezed between thumb and index finger, the fingers will create an indentation on the stimulus surface. The shape of this indentation, or rather the pressure distribution over the contact area, depends on the stimulus compliance. Therefore, this pressure distribution can be used as a cue for the perception of hardness. In this experiment, this cue was removed in order to study its effect. Note also that with a rigid surface, the size of the contact area between finger and surface depends on the force because of the deformation of the fingertip [10]. Therefore, also with a rigid surface, the size of the contact area can be used to determine the magnitude of the applied force. Thus, cutaneous information about the force is still present. However, in order to act as a cue for compliance perception, this information needs to be combined with kinesthetic displacement information. In the present experiment, without surface deformation, it is no longer possible to perceive compliance using only cutaneous cues.

\subsection{Method}

\subsubsection{Subjects}

Eight new subjects (five male) participated in Experiment 2. They ranged in age between 18 and 26 years and were all right-handed.

\subsubsection{Stimuli}

For this experiment, only the "thick" stimuli were used. On the flat surfaces of both sides of the stimuli, 0.5-mm-thick steel discs with a diameter of $40 \mathrm{~mm}$ were applied. These did not deform noticeably under the conditions of being squeezed by the subjects. In this way, a similar effect as the spring cells in [6] was attained. Due to the much larger area compared to the fingertip, the hardest set of stimuli were no longer compressible by the subjects, and were therefore not used. Instead, a new set of stimuli were fabricated that were even softer than the pure soft rubber stimulus. These consisted of a mixture of M 4500 silicone rubber and up to 15 percent (mass) of the silicone liquid AK 350 (Wacker Silicones), following Freyberger and Färber [4]. The more the silicone rubber was diluted with silicone liquid, the softer the stimulus. The new set was characterized in the same way as the original stimuli.

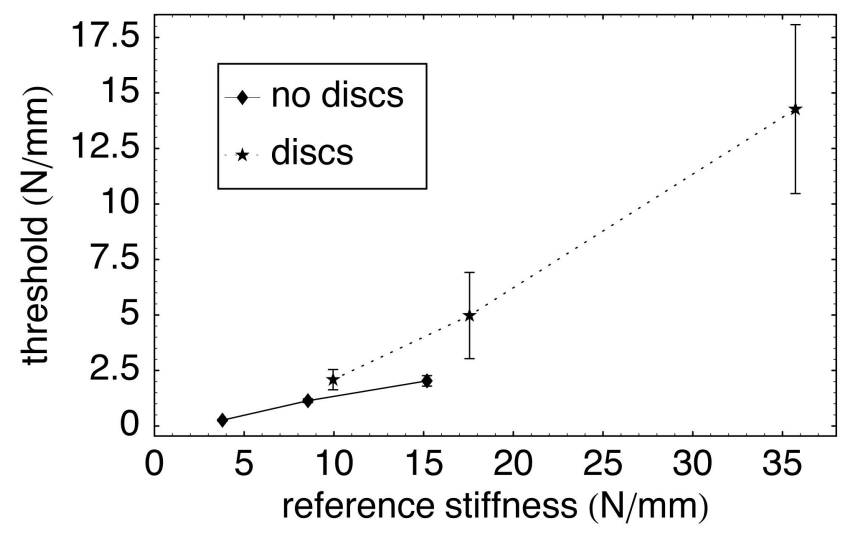

Fig. 6. Discrimination thresholds for the "thick" stimuli with and without the steel discs on the stimulus surfaces as a function of reference stiffness. The error bars indicate the standard error of the mean.

\subsubsection{Procedure}

The procedure was identical to that of Experiment 1, with, again, three compliance ranges, except this time only one type of stimulus was used. The three compliance ranges were mixed. The time taken for the 240 trials per subject ranged from 73 to 100 minutes.

\subsection{Results}

Because the force applied by the subject's fingers was now distributed over the whole surface of the stimulus, the data of this experiment were analyzed in terms of the stiffnesses that were measured using the flat surfaces. In Fig. 6, the thresholds are shown as a function of reference stiffness, averaged over subjects, together with the data for the "thick" stimuli from Fig. 5a. The data for the "no discs" case are expressed in terms of stiffness measured with the fingersized probes, while the data for the "discs" case are expressed in terms of stiffness measured with the flat surfaces. This ensures that the way the physical compliance is expressed resembles most the way the subjects experienced the stimuli. Since these are two different methods of measuring, the thresholds cannot be compared directly. However, the slopes of the lines that describe the relationship between threshold and reference hardness (Weber fractions) can be compared. The data measured with the discs can be described by a straight line with a slope of $0.5 \pm 0.3\left(R^{2}=0.996\right)$. Again, the standard deviation is derived from the spread in the slopes of the individual subjects. Although the overlap is only small due to the different hardness ranges, it is clear from the graph that the thresholds with discs are substantially higher than without. In fact, the slope of the straight line, and therefore, the Weber fraction, is increased by more than a factor of three.

\subsection{Discussion}

Without the cue of surface deformation present, the Weber fraction for hardness discrimination was found to be 0.5 . This is substantially higher than the value of 0.23 found by Jones and Hunter [17] and that of roughly 0.22 as found by Tan et al. [5] in their experiment with "roving displacement," both of which used rigid interfaces. The value is more than three times as high as the one found in Experiment 1, in which surface deformation cues were present. This once again shows the importance of direct contact with the compliant 
material for optimum discrimination of compliance. Srinivasan and LaMotte [6] had already shown that tactile cues dominate kinesthetic cues in the case of deformable surfaces, but the present experiments allow us to speculate about the relative importance of the available cues. Note that in the case with the metal discs, the contact area spread rate is only dependent on the compliance of the finger. Thus, in that case, it does not act as a cue for the perception of the stimulus compliance, but is a component of the force/displacement cue. The surface deformation cue and the force/displacement cue can be seen as two parallel information sources that are combined to produce the final perception, in this case, of the hardness of an object. In Experiment 2, with the discs, there was no surface deformation, so this cue provided little useful information about the compliance of the object. We can, therefore, suppose that the Weber fraction of 0.5 found in that experiment is based solely on the force/displacement ratio cue. If we know how the two cues are combined, we can calculate how much they contributed to the thresholds found in the first experiment. It has been shown that the combination of visual and haptic information is done in a statistically optimal fashion [18]. That is, using maximumlikelihood estimation, the variance of the combined information is minimized. This predicts that the threshold $\sigma_{A B}$ based on combined information from two information sources (A and $\mathrm{B}$ combined) is related to the thresholds based on single information sources $\left(\sigma_{A}\right.$ and $\left.\sigma_{B}\right)$ by

$$
\sigma_{A B}^{2}=\frac{\sigma_{A}^{2} \sigma_{B}^{2}}{\sigma_{A}^{2}+\sigma_{B}^{2}} .
$$

If we assume that this way of combining information, which Ernst and Banks [18] showed to hold for visual and haptic cues, also holds for the surface deformation and force/ displacement ratio cues, we can calculate the predicted threshold for an imaginary compliance discrimination experiment in which only surface deformation cues and no force/displacement ratio cues are available. The Weber fraction for this situation, $\sigma_{s}$, is given by

$$
\sigma_{s}=\frac{1}{\sqrt{\frac{1}{\sigma_{1}^{2}}-\frac{1}{\sigma_{2}^{2}}}} \approx 0.16 \pm 0.07
$$

where $\sigma_{1}=0.15 \pm 0.06$ is the Weber fraction from the "thick" condition in Experiment 1 and $\sigma_{2}=0.5 \pm 0.3$ is the Weber fraction from Experiment 2. We use Weber fractions instead of absolute threshold values, because we did not use the same reference stiffnesses in the two experiments, so we do not have absolute threshold values at a single stimulus intensity. Because of the linear behavior that is observed in the data, we can use the Weber fractions to estimate the threshold at any stimulus intensity. Thus, from the results of both experiments taken together, we predict that the Weber fraction for compliance discrimination based on surface deformation information alone is 0.16 , compared to the Weber fraction of 0.5 for force/displacement ratio information alone. This implies that the weights used for combining the information (proportional to the reciprocal of the square of the threshold) are $90 \pm 106$ percent surface deformation and $10 \pm 18$ percent force/displacement ratio. The high errors reported here are the consequence of the high spread in the individual thresholds in Experiment 2. Nevertheless, it can be concluded that the information provided by the surface deformation of a compliant object makes up about nine tenths of the information used to assess the hardness of that object, if the information is combined in a statistically optimal fashion.

From Experiments 1 and 2, we have seen how the discrimination thresholds are related to the stimulus configuration and compliance, and how big the relative roles of the available cues are. In Experiment 3, we investigated how the stimulus configuration influences the perception of compliance and what happens if the available cues appear to be in conflict.

\section{Experiment 3: Cross-Configuration COMPARISON}

The discrimination thresholds from Experiment 1 tell us something about the limits of human perception of compliance, but since all comparisons were done between stimuli of the same stimulus type, they cannot tell us about the influence of the stimulus configuration on compliance perception. For this purpose, we need to compare the perceived hardness of stimuli of different configurations with each other. Due to the presence of the rigid part in the sandwich stimuli, which is unknown to the subjects, a cue conflict arises. The subject has no reason to assume that the "sandwich" stimulus does not consist of silicone rubber all the way through, but the (local) surface deformation does not relate to the (global) force/displacement profile in the same way as is the case with a stimulus of the same thickness that is made completely from a single material. By studying how this conflict is resolved, we can check predictions that can be made based on the results from Experiments 1 and 2.

With three stimulus configurations, there are also three comparisons that can be made: "thick" versus "thin," "thick" versus "sandwich," and "sandwich" versus "thin." For these comparisons, we can look at how far stimuli should be apart in terms of physical compliance in order to be of equal perceived hardness. As we have seen before, this physical compliance can be expressed in different ways. For the comparison between "thick" and "thin," we expect no difference in physical compliance when it is expressed as the Young's modulus, that is, as a bulk property of the material. For stimuli with the same Young's modulus (made from the same material), the surface deformation will be similar, regardless of their thickness. In addition, the subject can correct for differences in the force/displacement profile by taking into account the differences in stimulus thickness. Thus, both cues can be used and lead to the same match between a "thick" and a "thin" stimulus.

The situation is different with the comparison between the "thick" and the "sandwich" stimuli: the subject feels stimuli with the same thickness, and therefore, expects matching stimuli to have the same surface deformation and the same force/displacement profile. However, due to the rigid part in the middle of the "sandwich" stimulus, this is impossible: stimuli of different types with the same surface deformation will have a different force/displacement 
profile and vice versa. The cues are in conflict and the subject has to make a choice in order to resolve this conflict. $\mathrm{He}$ or she can base his or her judgment entirely on one cue or entirely on the other cue. From Experiment 2, we learned that the surface deformation cue plays an important role, so if the subject chooses only one cue, we expect it to be the surface deformation cue. The third possibility is that a compromise is reached, and a match is made in which both the surface deformation and the force/displacement profiles are different. In that case, the Young's modulus of the "thick" stimulus will be higher than the "sandwich" stimulus with equal perceived hardness, while the force/ displacement ratio will be smaller. Based on the results from Experiment 2, we predict that in this case the difference in surface deformation will be smaller than the difference in force/displacement profile, since the surface deformation cue was found to be the most important.

The comparison between "sandwich" and "thin" will, from the subject's point of view, be the same as the "thick""thin" comparison, except also that, here, the cues are in conflict. In order for the three comparisons to be mutually consistent, we expect that this conflict is resolved in the same way as the "thick"-"sandwich" comparison. In order to test these predictions, another discrimination experiment was performed, in which subjects were repeatedly asked to select which of the two stimuli was made of the harder material, regardless of the stimulus thickness. By fitting a psychometric curve to the data with a free bias parameter $\mu$, we can measure which stimuli of the different configurations match in terms of perceived hardness. In this respect, the experiment can be considered a "guided" matching experiment, in which the subjects do not pick a matching stimulus themselves, but are guided toward such a match by subsequent discrimination trials.

\subsection{Method}

\subsubsection{Subjects}

Eight new subjects (five male), participated in this experiment. Their ages ranged from 17 to 29 years. All but one were right-handed. The left hander used his left hand for the experiment.

\subsubsection{Stimuli}

The stimuli from Experiment 1 were used. Each of the three types of stimulus was compared to the two other types, and for each of these six comparisons, two reference stimuli were selected: one located in the soft part of the range and one in the hard part. So, in total, there were 12 reference stimuli. These were chosen in such a way that their match was expected to fall in the range where the spacing of the test stimuli was the most dense, based on pilot experiments.

\subsubsection{Procedure}

For each of these 12 reference stimuli, a separate interleaved one-up/one-down staircase procedure was used. In this procedure, the reference stimulus was presented together with a test stimulus that was different from trial to trial. In the first trial, the test stimulus was at the soft end of the range. In the second trial, it was at the hard end of the range. In the third and subsequent trials, the test stimulus was chosen based on the response two trials ago: if the subject indicated that the test stimulus was made of the harder material, the next test stimulus would be one step softer, and vice versa. In this way, the perceived difference between test and reference became progressively smaller. This allowed for precise pinpointing of the shift in physical hardness that was associated with equal perceived hardness. Because the point of equal perceived hardness was alternately approached from two different directions, a biasing effect caused by the starting point of the staircase procedure was avoided. The positions of the test and reference stimuli were randomized for each trial. The order in which the 12 series were tested was different for each subject. Subjects were specifically instructed to select the stimulus that was made of the harder material, regardless of its thickness. Because it was important that subjects did not know that the "sandwich" stimuli had a rigid part in the middle, all stimuli were kept covered until the subject had donned the blindfold. Also, the stimuli were presented on a soft cloth in order to prevent the sound from the stimuli being put on the table revealing the presence of the hard part. Furthermore, adhesive tape was applied around the middle part of both the "thick" and the "sandwich" stimuli, so as to make them feel the same in case the subject accidentally brushed his or her fingers over this part of the stimuli. There were 25 trials for each of the 12 series, totaling 300 trials per subject. These were performed in two separate sessions that were either on different days or with at least 1 hour in between. The time taken ranged from 73 to 119 minutes.

\subsubsection{Analysis}

The percentage of times that the test stimulus was said to be made of the harder material was plotted as a function of the difference in physical hardness between the test and the reference stimulus. As mentioned before, this physical hardness can be expressed in different ways. In the analysis, we will use both the force/displacement ratio and the Young's modulus. An example is shown in Fig. 7. A psychometric curve of the form

$$
y=\frac{1}{2} \operatorname{erf}\left(\frac{x-\mu}{\sqrt{2} \sigma}\right)+\frac{1}{2}
$$

was fitted to the data to determine the Point of Subjective Equality (PSE). This PSE indicates the compliance of the test stimulus type needed to let it feel equally hard as the reference type. Due to the use of a staircase procedure, not all points were based on the same number of trials. Therefore, a maximum-likelihood method, which takes into account the number of trials a point is based on, was used as the fit procedure [19]. In this way, the shift in physical compliance necessary to produce equal perceived hardness was determined.

\subsection{Results}

In Fig. 8, the compliances that were found to be perceptually equal for the different stimulus configurations are connected by arrows. The matching was done in both directions for each comparison, and these appear to be consistent. The three comparisons are also mutually consistent. Using 


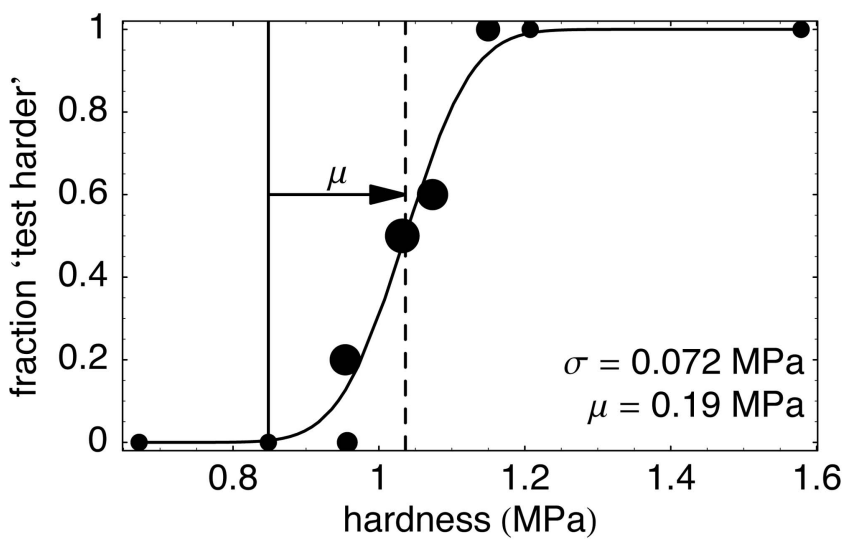

Fig. 7. Example of a psychometric curve with fitted function for the crossconfiguration experiment. The reference stimulus had a "sandwich" configuration, while the tests were of the "thick" type. In this example, the hardness is expressed as the Young's modulus of the material. The size of the dots is an indication of the number of trials the point is based on. The vertical solid line indicates the hardness of the reference stimulus. The dashed line indicates the position of the 50 percent point of the fitted curve. The difference between the compliances of the two types that are perceived to be of equal hardness $(\mu)$ is indicated.

$t$-tests, we assessed whether the compliances of two stimuli of perceptually equal hardness were significantly different. The analysis was done with the compliance expressed in different ways. With compliance expressed as the Young's modulus, the differences were significant for all comparisons except those between "thin" and "thick" (Fig. 8a). When compliance was expressed as the slope of the force/ displacement profile, the differences between "thin" and "thick" were significant, as were those between "thick" and "sandwich" (Fig. 8b). The difference between "thin" and "sandwich" was not significant.

\subsection{Discussion}

As predicted, the Young's moduli of the materials in the comparison between "thin" and "thick" were correctly matched, regardless of stimulus thickness (Fig. 8a). In the comparison between "thick" and "sandwich," a "thick" stimulus was matched with a physically significant softer "sandwich." Therefore, the cue conflict that was present in this case was not resolved by only taking into account the Young's modulus.

However, looking at the plot with compliance expressed as the slope of the force/displacement profile (Fig. 8b), we see that here, the comparison between "thick" and "sandwich" also yields a significant difference: a "thick" stimulus is matched with a significantly harder "sandwich." This means that the cue conflict was also not resolved by just attending to the force/displacement profile. In fact, the subjects seem to have made a compromise between keeping the surface deformation the same and keeping the force/ displacement profile the same. Comparing the middle panels of Figs. 8a and 8b, it seems that subjects have kept the surface deformation (Young's modulus) more constant than the force/displacement profile, although these quantities cannot be compared directly. A comparison can be made using the relative changes made in the two cues. For this purpose, we divide the absolute difference between the compliance of matching "sandwich" and "thick" stimuli by their mean compliance. We see that, on average, the Young's modulus differs 21 percent while the force/displacement ratio differs 38 percent. Subjects are more willing to compromise with the force/displacement ratio than with the Young's modulus.

(a)
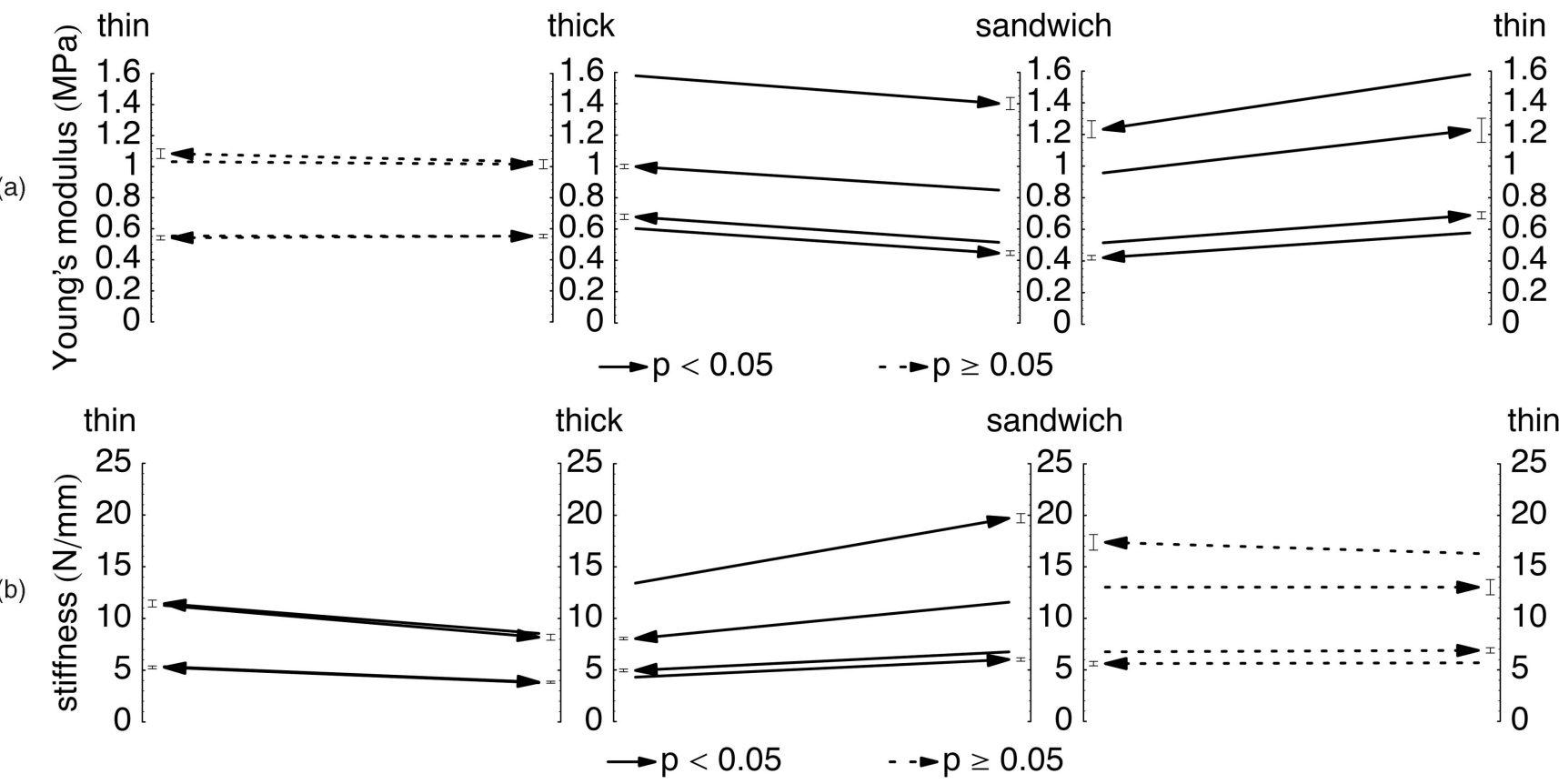

Fig. 8. Plots of the compliance of matched stimuli for the different configurations. The arrows connect the compliance of a reference stimulus to the matching compliance of a stimulus of a different type, averaged over subjects. The error bars indicate the standard error of the mean. Matches between compliances that are not significantly different ( $t$-test, $p \geq 0.05$ ) are indicated by dotted lines. (a) Compliance expressed as Young's modulus; (b) compliance expressed as the slope of the force/displacement profile as measured with the small probes. 


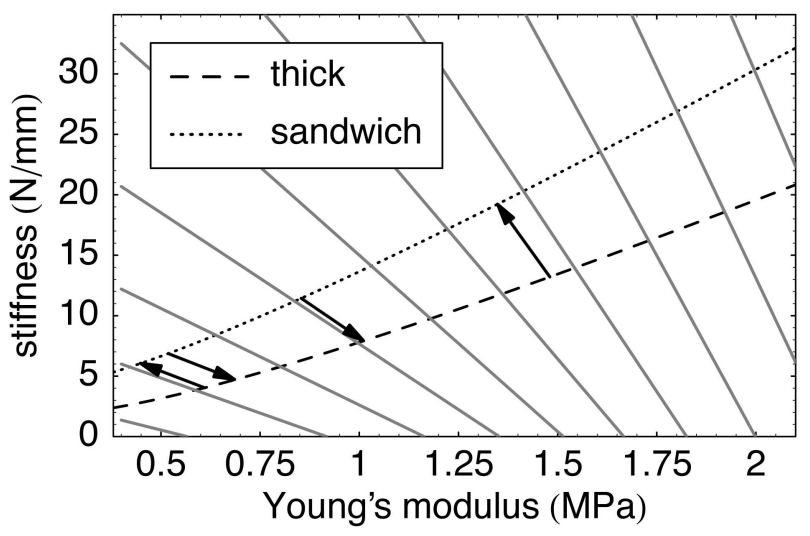

Fig. 9. Relationship between the slope of the force/displacement profile and the Young's modulus for two types of stimuli (dashed and dotted curves) based on fits to the measured data. The arrows indicate the matches made between these stimulus types. The gray lines illustrate possible curves of equal perceived hardness, based on the measurements.

This may be due to the fact that the perception of the Young's modulus, as a bulk material parameter, may be more dependent on cutaneous cues, while the force/displacement ratio may be more dependent on kinesthetic cues. The results would then be in line with those of Srinivasan and LaMotte [6] and Bicchi et al. [10], who showed that cutaneous cues dominated kinesthetic cues in softness perception.

Incidentally, in Fig. 8b, we see that the comparison between "thin" and "sandwich" does not show a difference that is significant at the 0.05 level. Due to the difference in thickness between these stimulus types, we have no reason to expect that the perceptually equal hardnesses should also have the same force/displacement profile. On the other hand, there is also no reason to expect that this should not be the case. Because the difference in physical stiffness between "thick" and "thin" stimuli of equal perceived hardness happens to be approximately equal to the difference between "thick" and "sandwich" stimuli of equal perceived hardness, and due to the consistency between the measurements, the difference between "thin" and "sandwich" turns out to be insignificant.

From the results of the comparison between stimuli of different thickness, we can conclude that subjects are able to match identical materials independent of thickness. They do this either by keeping the surface deformation the same or by scaling the force/displacement ratio according to the perceived stimulus thickness. When matching stimuli of equal thickness ("sandwich" and "thick"), subjects expect both the surface deformation and the force/displacement ratio to remain the same. When this turns out to be impossible, a compromise is made and a stimulus is selected that has a lower force/displacement ratio, although it consists of a material that has a higher Young's modulus or vice versa. This indicates that the subject does not ignore one cue or the other, but tries to take both of them into account. The way these two quantities are adjusted can be illustrated in a diagram that relates the slope of the force/displacement profile to the Young's modulus for these two types of stimuli; see Fig. 9. In the figure, arrows connect the locations of stimuli of different types that were perceived to be of equal hardness. The direction of these arrows depends on their location in the graph. To the direction of these arrows, a quadratic function was fitted. The direction derived from this function is indicated in the graph by gray lines that connect points of equal perceived hardness. Although they are drawn over the whole plotting range, it should be noted that the extrapolation may not be reliable over this whole range. The directions of these lines seem to indicate that for harder stimuli, where the lines are oriented more toward the vertical, it is more important to keep the surface deformation the same, while for the softer stimuli, where the lines are oriented more horizontally, subjects prefer to keep the force/ displacement ratio the same. This suggests that the importance of a cue depends on the stimulus compliance.

\section{General Discussion and Conclusions}

The first finding of this paper is that the Weber fraction for hardness discrimination is $\sim 15$ percent regardless of stimulus thickness or finger spread. This is substantially better than reported in [4], which is surprising since they used very similar materials. It is more comparable to the results reported in [2] and [3]. But, more importantly, the thresholds for the three stimulus configurations have the same linear dependence on the reference hardness. This means that thresholds did not increase when the finger span was increased for stimuli with the same Young's modulus. Thus, the accuracy of perceiving hardness of compliant materials probably does not depend on the accuracy of perception of the distance between the fingers. This implies that compliance can be perceived directly, without the need for (subconscious) calculation of the ratio of force increase and finger displacement. This is in line with the finding that tactile information is sufficient for discrimination of rubber specimens [6].

By combining the results from the first and the second experiment, and assuming that the information is combined in a statistically optimal fashion, it became clear that if information is combined in a statistically optimal fashion, a large majority of the information (90 percent) used in perceiving compliance originates from the perception of surface deformation. This means that measurements of hardness discrimination done using a rigid interface may underestimate the accuracy of the human perceptual system to a large extent. It also stresses the importance of including surface deformation information in haptic displays, which, up to now, are generally only able to display force information. A notable exception is the CASR display, which is able to simulate objects of different compliances by modifying the contact area spread rate [10].

Finally, the third experiment showed that the perceptual system, when assessing the compliance of a material, correctly takes into account the object thickness. When different cues (surface deformation and force/displacement ratio) appear to be in conflict, they can be interchanged to produce the same perception of hardness. That is, an object with a lower Young's modulus and a higher force/ displacement ratio can feel equally hard as another object with a higher Young's modulus and a lower force/ displacement ratio. This can be illustrated by means of lines of equal hardness in graph of force/displacement ratio 
versus Young's modulus. The angles of these lines tell us something about the relative importance of the different cues as a function of stimulus compliance.

In conclusion, humans are quite good at directly perceiving the compliance of a material. They do this for the most part by using information about the deformation of the surface, but the other cue of force/displacement ratio also influences the judgment, in particular, with softer materials.

\section{ACKNOWLEDGMENTS}

The authors wish to thank Dr. Niels Anten (Dept. of Biology) for the use of the Instron 5542. This work was supported by a grant from the Netherlands Organization for Scientific Research (NWO).

\section{References}

[1] R. Harper and S.S. Stevens, "Subjective Hardness of Compliant Materials," Quarterly J. Experimental Psychology, vol. 16, pp. 204215, 1964.

[2] G.W. Scott Blair and F.M.V. Coppen, "The Subjective Judgements of the Elastic and Plastic Properties of Soft Bodies; the 'Differential Thresholds' for Viscosities and Compression Moduli," Proc. Royal Soc. B, vol. 128, pp. 109-125, 1939.

[3] F.M.V. Coppen, "The Differential Threshold for the Subjective Judgement of the Elastic and Plastic Properties of Soft Bodies," British J. Psychology, vol. 32, no. 3, pp. 231-247, 1942.

[4] F.K.B. Freyberger and B. Färber, "Compliance Discrimination of Deformable Objects by Squeezing with One and Two Fingers," Proc. EuroHaptics '06, pp. 271-276, 2006.

[5] H.Z. Tan, N.I. Durlach, G.L. Beauregard, and M.A. Srinivasan, "Manual Discrimination of Compliance Using Active Pinch Grasp: The Roles of Force and Work Cues," Perception and Psychophysics, vol. 57, no. 4, pp. 495-510, 1995.

[6] M.A. Srinivasan and R.H. LaMotte, "Tactual Discrimination of Softness," J. Neurophysiology, vol. 73, no. 1, pp. 88-101, 1995.

[7] R.H. LaMotte, "Softness Discrimination with a Tool," J. Neurophysiology, vol. 83, no. 4, pp. 1777-1786, 2000.

[8] R.M. Friedman, K.D. Hester, B.G. Green, and R.H. LaMotte, "Magnitude Estimation of Softness," Experimental Brain Research, vol. 191, no. 2, pp. 133-142, 2008.

[9] D.A. Lawrence, L.Y. Pao, A.M. Dougherty, M.A. Salada, and Y. Pavlou, "Rate-Hardness: A New Performance Metric for Haptic Interfaces," IEEE Trans. Robotics and Automation, vol. 16, no. 4, pp. 357-371, Aug. 2000.

[10] A. Bicchi, E.P. Scilingo, and D. De Rossi, "Haptic Discrimination of Softness in Teleoperation: The Role of the Contact Area Spread Rate," IEEE Trans. Robotics and Automation, vol. 16, no. 5, pp. 496504 , Oct. 2000

[11] M.A. Srinivasan, G.L. Beauregard, and D.L. Brock, "The Impact of Visual Information on the Haptic Perception of Stiffness in Virtual Environments," Proc. ASME Dynamic Systems and Control Division, vol. 58, pp. 555-559, 1996.

[12] A. Lécuyer, S. Coquillart, A. Kheddar, P. Richard, and P. Coiffet, "Pseudo Haptic Feedback: Can Isometric Input Devices Simulate Force Feedback?" Proc. Virtual Reality Ann. Int'l Symp., pp. 83-90, 2000.

[13] X.D. Pang, H.Z. Tan, and N.I. Durlach, "Manual Discrimination of Force Using Active Finger Motion," Perception and Psychophysics, vol. 49 , no. 6 , pp. 531-540, 1991.

[14] N.I. Durlach, L.A. Delhorne, A. Wong, W.Y. Ko, W.M. Rabinowitz, and J. Hollerbach, "Manual Discrimination and Identification of Length by the Finger-Span Method," Perception and Psychophysics, vol. 46, no. 1, pp. 29-38, 1989.

[15] W.M. Bergmann Tiest and A.M.L. Kappers, "Kinaesthetic and Cutaneous Contributions to the Perception of Compressibility," Haptics: Perception, Devices, and Scenarios, M. Ferre, ed., pp. 255264, Springer, 2008.

[16] S. Coren, The Left-Hander Syndrome: The Causes and Consequences of Left-Handedness. Vintage Books, 1993.
[17] L.A. Jones and I.W. Hunter, "A Perceptual Analysis of Stiffness," Experimental Brain Research, vol. 79, no. 1, pp. 150-156, 1990.

[18] M.O. Ernst and M.S. Banks, "Humans Integrate Visual and Haptic Information in a Statistically Optimal Fashion," Nature, vol. 415, no. 6870, pp. 429-433, 2002.

[19] F.A. Wichmann and N.J. Hill, "The Psychometric Function: I. Fitting, Sampling, and Goodness of Fit," Perception and Psychophysics, vol. 63, no. 8, pp. 1293-1313, 2001.

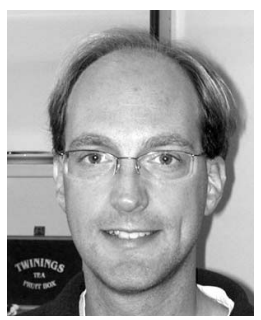

Wouter M. Bergmann Tiest received the MSc degree in experimental physics from Utrecht University, The Netherlands, in 1999. Until 2004, he was with the Netherlands Institute for Space Research, while pursuing the PhD from Utrecht University. He is currently a postdoctoral researcher in the Department of Physics and Astronomy of Utrecht University, working with the Human Perception Group of the Helmholtz Institute. His research interests include haptic searching, haptic counting, and haptic perception of volume, mass, and material properties such as roughness, thermal conductance, friction, compliance, and viscosity.

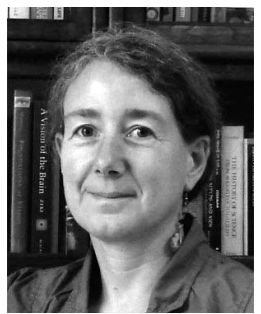

Astrid M.L. Kappers received the PhD degree from Eindhoven University of Technology. She studied experimental physics at Utrecht University, The Netherlands. Since 1989, she has been with the Department of Physics and Astronomy, Utrecht University. She was promoted to full professor in 2005. Her research is being implemented at the Helmholtz Institute. Her research interests include haptic and visual perception. In 2003, she won the prestigious $\mathrm{VICl}$ grant. She is member of the editorial boards of Acta Psychologica and Current Psychology Letters and associate editor of the IEEE Transactions on Haptics.

$\triangleright$ For more information on this or any other computing topic, please visit our Digital Library at www.computer.org/publications/dlib. 